\title{
APPLICATION OF COMPREHENSIVE FUZZY EVALUATION BASED ON STUDENT PARTICIPATION IN GENERAL EDUCATION COURSE EXAMINATION
}

\author{
Xuanze Zhao $^{1}{ }^{\square}$ and Xiaohui Zhou ${ }^{1}$ \\ ${ }^{1}$ Zhejiang University of Finance and Economics Dongfang College, Zhejiang, China
}
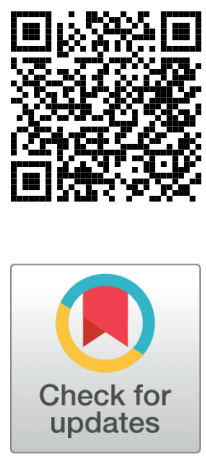

Received 5 May 2021

Accepted 20 May 2021

Published 31 May 2021

Corresponding Author

Xiaohui Zhou

DOI 10.29121/

granthaalayah.v9.i5.2021.3921

Funding: This research was funded by the Higher Education Teaching Reform Project of Zhejiang University of Finance and Economics Dongfang college, grant number 2021JK13.

Copyright: (C) 2021 The Author(s). This is an open access article distributed under the terms of the Creative Commons Attribution License, which permits unrestricted use, distribution, and reproduction in any medium, provided the original author and source are credited.

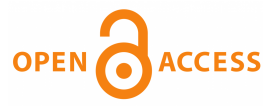

\section{ABSTRACT}

In view of the assessment methods of general education courses, an assessment and evaluation model is constructed, which consists of the usual results, material learning, cooperative discussion and classroom performance, and the final results, which are combined with on-site assessment and paper assessment. Introducing students to participate in the process assessment and onsite assessment of general education courses innovatively improves students' enthusiasm to participate in the general education class. In the process of empirical research, the order relation analysis method was firstly used to judge the importance of "homework summary, material learning, cooperative discussion, classroom performance ", etc., and then the weight coefficient was determined. Then the comprehensive evaluation method based on fuzzy mathematics was adopted. Homework summary, material learning, cooperative discussion, classroom performance review and field is not so easy to use, such as the exact scores show with the method of fuzzy evaluation, the process of fully reflect the students' learning process of a curriculum, and intensify students participate in class, let students participate in curriculum evaluation, can more active classroom and improve the effect of course.

Keywords: : Comprehensive Fuzzy Evaluation, Evaluation Model, Order Relation Analysis

\section{INTRODUCTION}

Under the background of the rapid development of higher education several years ago, new requirements have been put forward for curriculum assessment, especially for general education courses. The former single mode of "one exam for life" could not comprehensively evaluate students' learning from the aspects of learning, research, experimental practice and innovation, and students could not participate in the course evaluation, which to a large extent restricted and constrained students' 
learning and innovation ability as well as their enthusiasm for course participation. Therefore, we need to research and develop more scientific and reasonable assessment methods, assessment means and measures, strengthen learning process management and strictly process assessment. However, in order to show students' learning effect of this course, we can even have different assessment forms for the same subject. The way of assessment is the baton, we want to make the assessment really become an effective method and inspection way to promote students' learning, mobilize students' enthusiasm, and finally realize the teaching according to their aptitude and comprehensive education.

For the general course of literature and art and culture, mostly belongs to expand their horizons, cultivate appreciate the skills and the ability to discern value of course, we also generally final exam according to the different levels of students and interests adopted the on-site examination and assessment, as long as based on the general education curriculum content, not constrained by the inspection form, as long as is beneficial to show students' personal abilities, To integrate the understanding and thinking of such courses, for example, the on-site assessment can adopt the presentation methods that college students are good at, such as story speech, sketch interpretation, group debate and so on. As the usual results and on-site assessment criteria are open and easy to identify, we specially invite some students to participate in the assessment and evaluation, and even the on-site assessment students themselves can participate in the evaluation, which greatly enhances the students' enthusiasm for the participation in the class.

\section{THE EVALUATION MODEL OF GENERAL EDUCATION COURSE AND THE BASIC PRINCIPLE OF FUZZY COMPREHENSIVE EVALUATION}

\subsection{MULTI-ATTRIBUTE ASSESSMENT AND EVALUATION MODEL FOR GENERAL EDUCATION COURSES}

The overall score of the multi-assessment of general education courses is generally composed of the usual score and the final score, and the usual score generally includes assignment and summary, material study, cooperation discussion, classroom performance and so on several aspects as evaluation indicators, and every aspect and has a number of specific elements, usually several times such as assignments 1, 2, 3, and so on as a secondary indexes, and the final exam we also according to the different levels of students and adopted the on-site examination and assessment, As long as it is based on the content of the general education course, it is not restricted to the form of assessment. For example, the on-site assessment can adopt the presentation methods that college students are good at, such as story speech, sketch interpretation, group debate and so on. As the usual results and on-site assessment criteria are open and easy to identify, students are specially invited to partici- 
pate in the assessment and evaluation, and even students themselves can participate in the evaluation, which greatly enhances the enthusiasm of students to participate in the class. On this basis, we can establish the following multi-attribute assessment and evaluation model for general education courses (see Figure 1 ).

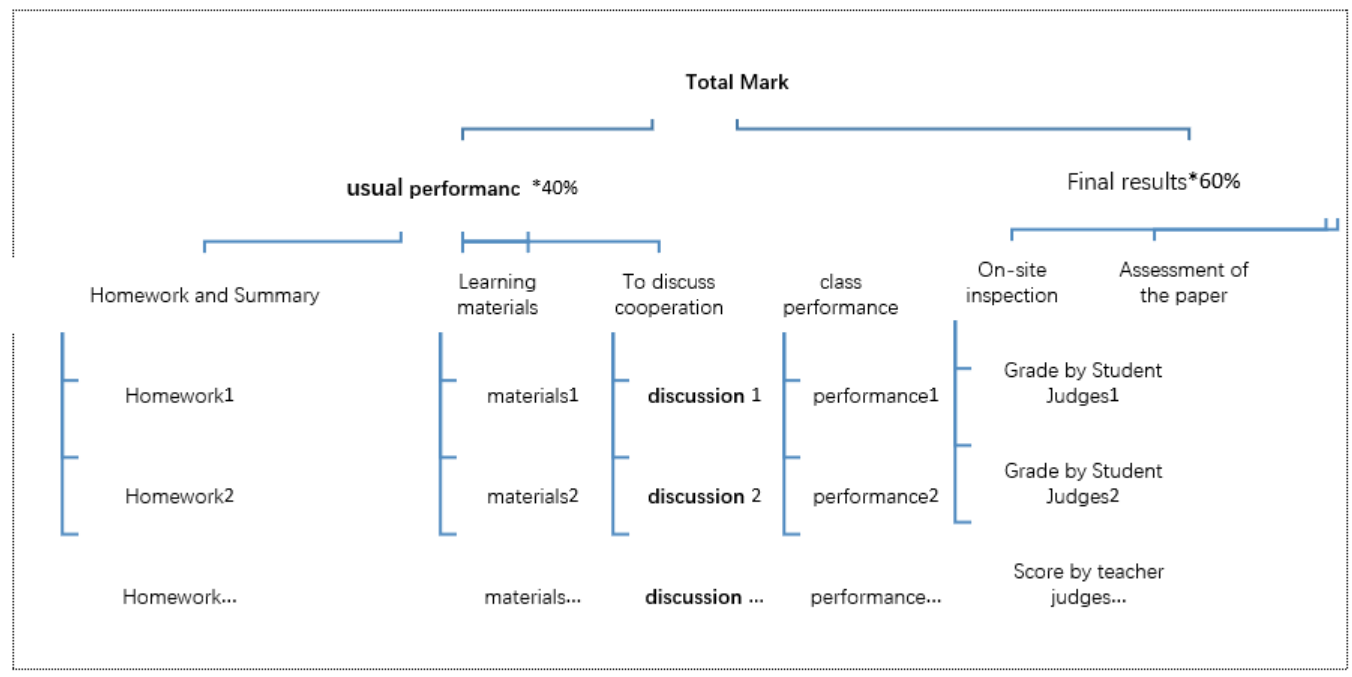

Figure 1 Multi-attribute assessment and evaluation model for general education courses

\subsection{TO DETERMINE THE WEIGHT OF THE ORDER RELATION ANALYSIS AND FUZZY COMPREHENSIVE EVALUATION OF THE BASIC IDEAS}

Firstly, the order relation analysis method Yajun (2007)is used to determine the order relation, and then the comparative judgment of the relative importance is given to obtain the weight coefficient. In determining the job summary, material study, cooperation discussion, classroom performance, etc. The content of the evaluation index and weight ratio, according to the fuzzy membership function indexes describing boundaries, constructing fuzzy evaluation matrix, at last, through fuzzy evaluation matrix and the evaluation grades of multilayer composite computing into evaluation object of the evaluation result Li (2011b).

\subsection{THE BASIC PRINCIPLE AND METHOD OF FUZZY COMPREHENSIVE EVALUATION}

\subsubsection{THE WEIGHT OF EVALUATION INDEX IS DETERMINED BY USING THE ANALYSIS OF ORDER RELATION}

The eigenvalue method is generally used to determine the index weight, because the eigenvalue method looks very beautiful and the calculation method is not difficult, but it is based on the fact that the judgment matrix is consistent matrix, and the judgment matrix established in specific applications is often not consistent matrix; Then the only criterion for solving the problem correctly by eigenvalue method is the 
random consistency ratio of the judgment matrix, which is powerless in this case. In addition, the eigenvalue method has a large amount of calculation, and according to the results of psychological experiments, when the number of elements compared exceeds 9, the judgment is not accurate, that is, the eigenvalue method cannot be used, so at this time, we can use the order relation analysis method (G1method) Yajun (2007).

\section{Determine the order relation}

According to the degree of importance, if the evaluation index $x_{i}$ is greater than (or not less than) $x_{j}$ relative to some evaluation standard, we denote it as $x_{i} \succ x_{j}$.Then evaluation index $x_{1}, x_{2}, \cdots, x_{m}$ compared with a certain evaluation standard is relation between $x_{1}^{*} \succ x_{2}^{*} \succ \cdots \succ x_{m}^{*}$, we call it the evaluation index $x_{1}, x_{2}, \ldots, x_{m}$ according to " $\succ$ " established the order relation Li (2011b) .Here, $x_{i}^{*}$ denoted the ith evaluation index(i=1,2,...,m).

Evaluation index set $\left\{x_{1}, x_{2}, \ldots, x_{m}\right\}$ order relation algorithm:

For $(\mathrm{i}=\mathrm{m}$ to 1$)$

most important(m) / In the index set $\left\{x_{1}, x_{2}, \ldots, x_{m}\right\}$, the expert chooses the most important index and denotes it as $x_{i}^{*} /$

After m-1 selection, the expert can mark the last most important indicator, denoted as $x_{m}^{*}$

\section{Give a comparative judgment of relative importance}

Suppose that the importance ratio of evaluation index $x_{k-1}$ to $x_{k}$ by experts is denoted as:

$$
r_{k}=\frac{x_{k-1}}{x_{k}}(k=m, m-1, \ldots, 3,2)
$$

When $m$ is large, $r_{m}$, determined by the order relation, is equal to 1 . In general, the assignment of $r_{k}$ is shown in the following Table 1 Liu and Zhong (2013):

Table $1 r_{k}$ assignment reference table

\begin{tabular}{ll}
\hline$r_{k}$ & Explanation \\
1.0 & The index $x_{k-1}$ is of equal importance to the comparison of the index \\
1.2 & The index $x_{k-1}$ is slightly more important than the index $x_{k}$ \\
1.4 & The index $x_{k-1}$ is obviously more important than the index $x_{k}$ \\
1.6 & The index $x_{k-1}$ is strongly more important than the index $x_{k}$ \\
1.8 & The index $x_{k-1}$ is extremely more important than the index $x_{k}$ \\
\hline
\end{tabular}

Theorem 1.1.1 If $x_{1}, x_{2}, \ldots, x_{m}$ has the order relation, then $r_{k-1}$ and $r_{k}$ will meet $r_{k-1}>\frac{1}{r_{k}}(k=m, m-1, m-2, \ldots, 3,2)$

Calculation of weight coefficient $w_{k}$

Theorem 1.1.2 If the expert gives a rational assignment of $r_{k}$ that satisfies the relationship

$$
r_{k-1}>\frac{1}{r_{k}}(k=m, m-1, m-2, \ldots, 3,2) \text {,so } w_{m}=\left(1+\sum_{k=2}^{m} \prod_{i=k}^{m} r_{i}\right)^{-1},
$$


then

$$
w_{k_{-1}}={ }_{r_{k}} w_{k},(k=m, m-1, m-2, \ldots, 3,2)
$$

\subsubsection{THE DETERMINATION OF THE MEMBERSHIP MATRIX R}

The evaluation factor set $U$ is the first-level indicator of homework summary, material learning, cooperative discussion, classroom performance, etc. Then each factor set contains several sub-factors, namely the second-level indicator. The evaluation level theory field $V$ is the fuzzy evaluation vector of fuzzy evaluation, and it is the concentrated embodiment of "fuzzy" in fuzzy evaluation. In fact, homework summary, material learning, cooperative discussion, classroom performance and other evaluations are difficult to be accurately measured by specific scores, but can only be measured by fuzzy concepts such as "excellent", "good", "medium", "poor", " very poor" and so on. Fuzzy comprehensive evaluation can solve the quantification of qualitative problems very well Zhanming and Yongjiang (2014). The evaluation index can be quantified into five grades: $v_{1}$ means "excellent", $v_{2}$ means "good", $v_{3}$ means "medium", $v_{4}$ means "poor", and $v_{5}$ means "very poor". Then the evaluation set $V=\left\{v_{1}, v_{2}, v_{3}, v_{4}, v_{5}\right\}$ is the evaluation level theory domain.The relationship between evaluation factors and evaluation grades, namely the fuzzy relationship from $U$ to $V$, can be expressed by the fuzzy evaluation matrix $R$ as follows:

$$
R=\left(r_{i j}\right)_{n \times m}=\left[\begin{array}{cccc}
r_{11} & r_{12} & \ldots & r_{1 m} \\
r_{21} & r_{22} & \ldots & r_{2 m} \\
\ldots & \ldots & \ldots & \ldots \\
r_{n 1} & r_{n 2} & \ldots & r_{n m}
\end{array}\right]
$$

Element $r_{i j}(\mathrm{i}=1,2, \ldots, \mathrm{n} ; \mathrm{j}=1,2, \ldots, \mathrm{m})$ in $R$ represents the membership degree Emrouznejad and Ho (2017) rated as $v_{j}$ based on the evaluation factor $u_{i}$, that is, the evaluation result corresponding to each evaluation index, in other word, the statistical result corresponding to the five grades of this index. It is generally the proportion in specific applications.

\subsubsection{OPERATION AND RESULT OF FUZZY JUDGMENT MATRIX}

For every being measured course, first to the primary index factors "homework summary, material learning, discuss cooperation and classroom performance" of the fuzzy evaluation matrix $R_{i}$ matrix, by the formula (2) can be primary index factors $U_{i}$ for membership vector $V_{i}$ comment set $V, B_{i}=A_{i} \circ R_{i}$ (० said it's operation in fuzzy mathematics, equivalent to two matrix multiplication), Then the fuzzy matrix 
operation is carried out on $R$ to obtain the membership vector of target level index (first-level index) $U$ to comment set $V$ Munier and Hontoria (2021).

$$
B=A \circ R
$$

$$
F=W \circ D
$$

In this case, the sum of membership degrees is 1; if not, normalization is carried out Li (2011a).The elements in the result vector $F$ are taken as the weight of the corresponding elements in the evaluation set, and finally a specific value is obtained by using the weighted average algorithm, that is, the final score of students is assessed by using fuzzy evaluation.

\section{EMPIRICAL RESEARCH}

\subsection{THE WEIGHT WAS OBTAINED BY ORDER RELATION ANALYSIS}

In the multi-attribute evaluation model Figure 1 of the general education curriculum there are four evaluation index $x_{1}, x_{2}, x_{3}, x_{4}$, according to the Equation (1) expert thinks $x_{1}, x_{2}, x_{3}, x_{4}$ with order relation between $x_{1} \succ x_{2} \succ x_{4} \succ x_{3} \Rightarrow x_{1}^{*} \succ x_{2}^{*} \succ$ $x_{3}^{*} \succ x_{4}^{*}$,It can be obtained by Equation (2)

$r_{2}=\frac{w_{1}^{*}}{w_{2}^{*}}=1.6, r_{3}=\frac{w_{2}^{*}}{w_{3}^{*}}=1.2, r_{4}=\frac{w_{3}^{*}}{w_{4}^{*}}=1.4$

We can draw $r_{2} r_{3} r_{4}=2.688, r_{3} r_{4}=1.680, r_{4}=1.400$

$$
\begin{gathered}
r_{2} r_{3} r_{4}+r_{3} r_{4}+r_{4}=5.768 \\
w_{4}^{*}=(1+5.768)^{-1}=0.1477 \\
w_{3}^{*}=w_{4}^{*} * r_{4}=0.1477 * 1.400=0.2068 \\
w_{2}^{*}=w_{3}^{*} * r_{3}=0.2068 * 1.200=0.2482 \\
w_{1}^{*}=w_{2}^{*} * r_{2}=0.2482 * 1.600=0.3973
\end{gathered}
$$

Therefore, the weight coefficients of evaluation indexes $x_{1}, x_{2}, x_{3}$ and $x_{4}$ are respectively: 


$$
w_{1}=w_{1}^{*}=0.3973, w_{2}=w_{2}^{*}=0.2482 w_{3}=w_{4}^{*}=0.1477 w_{4}=w_{3}^{*}=0.2068
$$

In other words, the weight coefficients of homework summary, material learning, cooperative discussion and class performance are:(0.3973,0.2482,0.1477,0.2068),For convenience of calculation, we can take $(0.40,0.25,0.15,0.20)$ respectively.

\subsection{FUZZY EVALUATION PROCESS IN WHICH STUDENTS PARTICIPATE}

In fact, homework summary, material learning, cooperative discussion and class performance are difficult to be precise with specific scores, but can only be expressed with fuzzy concepts such as "excellent", "good", "medium", "poor", "very poor", and so on. Fuzzy comprehensive evaluation can solve the quantification of qualitative problems very well. We can determine the results of the homework summary according to the steps of the fuzzy evaluation method:

We can select factor sets (such as homework summary) $U=\left\{u_{1}, u_{2}, u_{3}, u_{4}, u_{5}, u_{6}, u_{7}, u_{8}\right\}$, $u_{1}$ represents the first homework summary in the rain class, $u_{2}$ represents the second homework summary in the rain class, $u_{3}$ represents the third homework summary in the rain class, and so on. $u_{8}$ represents the eighth homework summary of rain class.Then the evaluation set $V=\left\{v_{1}, v_{2}, v_{3}, v_{4}, v_{5}\right\}$, where $v_{1}$ means "excellent", $v_{2}$ means "good", $v_{3}$ means "medium", $v_{4}$ means "poor", and $v_{5}$ means "very poor", and the corresponding percentage scale score ranges are respectively: $90 \leq v_{1} \leq 100,80$ $\leq v_{2} \leq 89,70 \leq v_{3} \leq 79,60 \leq v_{4} \leq 69,0 \leq v_{5} \leq 59$

Based on the above analysis of evaluation indicators and methods, we investigated a student of a general education course and conducted an empirical evaluation of his performance in this course. In order to ensure the fairness of students' participation in evaluation, teachers were assigned to temporary groups to participate in on-site assessment, evaluation and grading. Ten students from one group were selected to evaluate the student's performance. $E=(5,4,3,2,1)^{T}$ :

The evaluation matrix of "homework summary" can be obtained from Equation (3), (4), (5)

$$
R_{1}^{\circ} E=\left[\begin{array}{ccccc}
0.6 & 0.3 & 0.1 & 0 & 0 \\
0.3 & 0.3 & 0.4 & 0 & 0 \\
0.5 & 0.5 & 0 & 0 & 0 \\
0.6 & 0.4 & 0 & 0 & 0 \\
0.7 & 0.2 & 0.1 & 0 & 0 \\
0.6 & 0.2 & 0.2 & 0 & 0 \\
0.5 & 0.4 & 0.1 & 0 & 0 \\
0.6 & 0.3 & 0.1 & 0 & 0
\end{array}\right]\left[\begin{array}{l}
5 \\
4 \\
3 \\
2 \\
1
\end{array}\right]=\left[\begin{array}{l}
4.5 \\
3.9 \\
4.5 \\
4.6 \\
4.6 \\
4.4 \\
4.5 \\
4.5
\end{array}\right] \underset{\exists-1}{\longrightarrow}\left[\begin{array}{l}
0.1268 \\
0.1099 \\
0.1268 \\
0.1296 \\
0.1296 \\
0.1239 \\
0.1268 \\
0.1268
\end{array}\right]^{T}
$$

$$
\left[\begin{array}{l}
0.1268 \\
0.1099 \\
0.1268 \\
0.1296 \\
0.1296 \\
0.1239 \\
0.1268 \\
0.1268
\end{array}\right]^{T}\left[\begin{array}{ccccc}
0.6 & 0.3 & 0.1 & 0 & 0 \\
0.3 & 0.3 & 0.4 & 0 & 0 \\
0.5 & 0.5 & 0 & 0 & 0 \\
0.6 & 0.4 & 0 & 0 & 0 \\
0.7 & 0.2 & 0.1 & 0 & 0 \\
0.6 & 0.2 & 0.2 & 0 & 0 \\
0.5 & 0.4 & 0.1 & 0 & 0 \\
0.6 & 0.3 & 0.1 & 0 & 0
\end{array}\right]=\left[\begin{array}{lllll}
0.5546 & 0.3256 & 0.1197 & 0.0000 & 0.0000
\end{array}\right]=B_{1}
$$




Similarly, $\quad R_{2}=\left[\begin{array}{ccccc}0.5 & 0.2 & 0.2 & 0.1 & 0 \\ 0.4 & 0.4 & 0.2 & 0 & 0 \\ 0.4 & 0.3 & 0.3 & 0 & 0 \\ 0.4 & 0.3 & 0.2 & 0.1 & 0 \\ 0.5 & 0.4 & 0.1 & 0 & 0 \\ 0.4 & 0.3 & 0.2 & 0.1 & 0\end{array}\right]$

We can draw $B_{2}=\left(\begin{array}{lllll}0.4326 & 0.3067 & 0.2265 & 0.0494 & 0.0000\end{array}\right)$ $R_{3}=\left[\begin{array}{ccccc}0.4 & 0.5 & 0.1 & 0 & 0 \\ 0.5 & 0.3 & 0.2 & 0 & 0 \\ 0.3 & 0.4 & 0.3 & 0 & 0 \\ 0.3 & 0.5 & 0.2 & 0.1 & 0\end{array}\right]$ We can draw $B_{3}=\left[\begin{array}{lllll}0.3763 & 0.4254 & 0.1982 & 0.0254 & 0.0000\end{array}\right]$ We can draw $B_{3}=\left(\begin{array}{lllll}0.3763 & 0.4254 & 0.1982 & 0.0254 & 0.0000\end{array}\right)$ $R_{4}=\left[\begin{array}{ccccc}0.4 & 0.4 & 0.1 & 0.1 & 0 \\ 0.3 & 0.3 & 0.4 & 0 & 0 \\ 0.5 & 0.4 & 0.1 & 0 & 0 \\ 0.4 & 0.3 & 0.2 & 0.1 & 0 \\ 0.5 & 0.2 & 0.3 & 0 & 0\end{array}\right]$ We can draw $B_{4}=\left[\begin{array}{lllll}0.4224 & 0.3215 & 0.2166 & 0.0395 & 0.0000\end{array}\right]$

We can draw $B_{4}=\left(\begin{array}{lllll}0.4224 & 0.3215 & 0.2166 & 0.0395 & 0.0000\end{array}\right)$

Students participating in the assessment by the upper can by the overall fuzzy evaluation of the course performance

$$
\begin{aligned}
& \mathrm{B}=\mathrm{A}^{\circ} \mathrm{R}=\mathrm{A}^{\circ}\left[\begin{array}{l}
B_{1} \\
B_{2} \\
B_{3} \\
B_{4}
\end{array}\right]=(0.40,0.25,0.15,0.20)\left[\begin{array}{ccccc}
0.5546 & 0.3256 & 0.1197 & 0 & 0 \\
0.4326 & 0.3067 & 0.2265 & 0.0494 & 0 \\
0.3763 & 0.4254 & 0.1982 & 0.0254 & 0 \\
0.4224 & 0.3215 & 0.2166 & 0.0395 & 0
\end{array}\right] \\
& =\left(\begin{array}{lllll}
0.4709 & 0.3350 & 0.1776 & 0.0241 & 0.0000
\end{array}\right)
\end{aligned}
$$

According to the above values of "excellent", "good", "medium", "poor" and "poor" according to the median of the assigned areas, they are respectively 95 points, 85 points, 75 points, 65 points and 30 points. Then the student's usual score of this course is 88.0946.

In the same way, the on-site assessment in the final exam is open to all the students who have chosen courses, and the scoring standard is also open. Therefore, in order to improve everyone's participation in the course, five student judges are set. If the student judges themselves participate in the on-site assessment, the students who participate in the on-site assessment will not evaluate themselves. Dissertation assessment does not necessarily accurate, because the student for the inspection process students don't participate in the evaluation, finally we participate in 
the evaluation grades of students and teachers for the curriculum evaluation results according to the 4:6 included in the total score, in accordance with the requirements of the school, grades and final grade proportion for 4:6, if a student grades to just calculate 88.0946 above, The final assessment method is on-site assessment. The result obtained by the five student judges from the comprehensive fuzzy evaluation is 89.4324 , and the result given by the teacher is 92 , so the student's total evaluation result is $88.0946 * 0.4+(89.4324 * 0.4+92 * 0.6) * 0.6=89.8216$

\section{CONCLUSION}

The assessment of general education courses that students participate in improves the enthusiasm of students to participate in general education classes. The evaluation model is constructed, which is composed of homework summary, material learning, cooperative discussion and classroom performance, and the final result which is combined with on-site assessment and paper assessment, and the evaluation mechanism of fuzzy evaluation is introduced. The results obtained in this way can not only fully reflect the process of students' learning general courses, but also increase the intensity of students' participation in the class and let some students participate in the course evaluation, which can more active in the class and improve the learning effect of the course.

\section{REFERENCES}

Emrouznejad, A., \& Ho, W. (2017). Fuzzy Analytic Hierarchy Process. In and others (Ed.), Analytic Hierarchy Process and Fuzzy Set Theory (p. 10). https://doi.org/10.1201/ 9781315369884-1

Li, J. Y. (2011a). Research and Thinking on the Evaluation Standard of Mathematics Teaching[J]. Journal of Mathematics Education, 5, 24-27.

Li, J. Y. (2011b). Research and Thinking on the Evaluation Standard of Mathematics Teaching[J]. Journal of Mathematics Education, 5, 24-27.

Liu, Z., \& Zhong, J. (2013). Study on Nursing Clinical Teachers' Comprehensive Quality Evaluation Model on the Basis of Fuzzy Mathematics. Research Journal of Applied Sciences, Engineering and Technology, 4, 3582-3585.

Munier, N., \& Hontoria, E. (2021). Uses and Limitations of the AHP Method A NonMathematical and Rational Analysis., 2, 43-45.

Yajun, G. (2007). Theory, method and application of comprehensive evaluation. science press (in chinese).

Zhanming, L., \& Yongjiang, Z. (2014). Vector "membership" based fuzzy controller structure optimization. Computer Engineering and Applications. 